\title{
Radio Observations of the Pulsar Wind Nebula HESS J1303-631 Field of View with ATCA
}

\author{
lurii Sushch* \\ Centre for Space Research, North-West University, Potchefstroom 2520, South Africa \\ Astronomical Observatory of Ivan Franko National University of L'viv, vul. Kyryla i Methodia, \\ 8, L'viv 79005, Ukraine \\ E-mail: iurii.sushch@nwu.ac.za
}

Igor Oya

DESY Zeuthen, Platanenallee 6, D-15738 Zeuthen, Germany

\section{Ullrich Schwanke}

Institut für Physik, Humboldt-Universität zu Berlin, Newtonstr. 15, D 12489 Berlin, Germany

\section{Simon Johnston}

Australia Telescope National Facility, CSIRO, PO Box 76, Epping, NSW 1710, Australia

\section{Matthew L. Dalton}

Active Space Technologies GmbH, Carl Scheele Strasse 14, 12489 Berlin, Germany

\begin{abstract}
Based on its enregy-dependent morphology the initially unidentified very high energy (VHE; $E \gtrsim 100 \mathrm{GeV}$ ) gamma-ray source HESS J1303-631 was recently associated with the pulsar PSR J1301-6305. Subsequent detection of X-ray and GeV counterparts also supports the identification of the H.E.S.S. source as evolved pulsar wind nebula (PWN). We report here on recent radio observations of the PSR J1301-6305 field of view (FOV) with ATCA dedicated to search for the radio counterpart of this evolved PWN. Observations at $5.5 \mathrm{GHz}$ and $7.5 \mathrm{GHz}$ do not reveal any extended emission associated with the pulsar. The analysis of the archival $1.384 \mathrm{GHz}$ and $2.368 \mathrm{GHz}$ data also does not show any significant emission. The $1.384 \mathrm{GHz}$ data reveal a hint of an extended shell-like emission in the PSR J1301-6305 FOV which might be a supernova remnant. We discuss the implications of the non-detection at radio wavelengths on the nature and evolution of the PWN as well as the possibility of the SNR candidate being the birth place of PSR J1301-6305.
\end{abstract}

The 34th International Cosmic Ray Conference,

30 July- 6 August, 2015

The Hague, The Netherlands

\footnotetext{
* Speaker.
} 


\section{Introduction}

HESS J1303-631 is one of the most prominent examples of the so-called very high energy (VHE; $E>100 \mathrm{GeV}$ ) $\gamma$-ray "dark" sources, those which were detected in the VHE band but did not have counterparts at other energy bands. It was discovered in 2005 (1) but the nature of the source was unclear until 2012, when a detailed study of the energy-dependent morphology provided evidence of the association with the pulsar PSR J1301-6305 (2). With the increase of the energy threshold a very extended emission region $\left(\sim 0.4^{\circ} \times 0.3^{\circ}\right.$ at the $(0.84-2) \mathrm{TeV}$ band $)$ of VHE $\gamma$-rays "shrinks" towards the position of the pulsar at $E>10 \mathrm{TeV}$. Such an energy-dependent morphology is expected for ancient pulsar wind nebulae (PWNe), which feature several populations of relativistic electrons generated by the pulsar. Young electrons located close to the pulsar are not cooled yet and, thus, very energetic. These energetic electrons generate the VHE emission around the pulsar via Inverse Compton (IC) scattering on the cosmic microwave background photons (CMB). Older, cooled down, lower energy electrons might be spread farther away from the pulsar for several reasons (e.g. proper motion of the pulsar which causes that older particles are left behind and/or particle diffusion) but they can still produce $\gamma$-rays via IC scattering, however at lower energies than the young electrons.

The association of HESS J1303-631 with the pulsar is further supported by the detection of its X-ray counterpart with XMM-Newton (2). The size of the X-ray PWN is much smaller than the size of the VHE source, extending $2^{\prime}-3^{\prime}$ from the pulsar position towards the center of the VHE $\gamma$-ray emission region. The much smaller size of the X-ray emitting region can be explained by an effective synchrotron cooling of older electrons to energies too low to generate synchrotron emission in the X-ray energy range and/or due to the decreasing of the magnetic field strength in the PWN with time (see e.g. 3). The tail-like extension of the X-ray source might be an indication of the proper motion direction of the pulsar triggering speculations about its possible birth-place (2).

A careful analysis of archival data from the Parkes-MIT-NRAO (PMN) survey at $4.85 \mathrm{GHz}$ (4) revealed also a hint of radio emission at the pulsar position with size comparable to the X-ray emission region. Data analysis showed a $\sim 3 \sigma$ feature with a peak flux of $0.03 \mathrm{Jy} / \mathrm{beam}$ which is at the detection limit of the survey (2). This hint of a radio counterpart of HESS J1303-631 triggered new dedicated observations with the Australian Telescope Compact Array (ATCA), which were conducted in September 2013. Results of these observations are presented in this paper.

Recently, the counterpart of HESS J1303-631 was finally detected at GeV energies with Fermi-LAT (5). The source is contaminated by the emission of the nearby Supernova remnant (SNR) Kes 17, but it is clearly seen above $31 \mathrm{GeV}$. The emission region of the $\mathrm{GeV}$ counterpart of HESS J1303-631 is as expected larger than the TeV source, but the morphology of the emission region is very similar and features an extension in the same direction as the $\mathrm{TeV}$ source.

\section{Observations and Data Analysis Results}

\subsection{Observations and Data Description}

The ATCA observations of the field of view (FOV) around PSR J1301-6305 were conducted on September 5th, 2013. Observations were performed with the 1.5A configuration of the array at 
Table 1: Details of the ATCA data of the HESS J1303-631 FoV analysed in this paper

\begin{tabular}{cccccc}
\hline \hline Date & Right Ascention & Declination & Time, [min] & Array & Frequencies, $[\mathrm{MHz}]$ \\
\hline & & & & & \\
$2013-$-Sep-05 & $13^{\mathrm{h}} 2^{\mathrm{m}} 10.00^{\mathrm{s}}$ & $-63^{\circ} 5^{\prime} 34.80^{\prime \prime}$ & 2626.7 & $1.5 \mathrm{~A}$ & 5500,7500 \\
$2006-$-Oct-25 & $13^{\mathrm{h}} 3^{\mathrm{m}} 0.40^{\mathrm{s}}$ & $-63^{\circ} 11^{\prime} 11.55^{\prime \prime}$ & 433.8 & EW352 & 1384,2368 \\
$2007-$ Mar-13 & $13^{\mathrm{h}} 3^{\mathrm{m}} 0.40^{\mathrm{s}}$ & $-63^{\circ} 11^{\prime} 11.55^{\prime \prime}$ & 618.1 & $750 \mathrm{D}$ & 1384,2368 \\
$2007-$ Apr-24 & $13^{\mathrm{h}} 3^{\mathrm{m}} 0.40^{\mathrm{s}}$ & $-63^{\circ} 11^{\prime} 11.55^{\prime \prime}$ & 651.8 & $1.5 \mathrm{C}$ & 1384,2368 \\
\hline
\end{tabular}

5.5 and 7.5 GHz frequencies and centered at $\alpha=13^{\mathrm{h}} 02^{\mathrm{m}} 10.0^{\mathrm{s}}, \delta=-63^{\circ} 05^{\prime} 34.8^{\prime \prime}(\mathrm{J} 2000.0)$. The total time of observations was 2626.7 minutes. Primary and secondary calibrators were $1934-638$ and $1352-63$, respectively. All the details of the collected data are listed in Table 1.

In this paper we also considered archival ATCA data obtained during observations of HESS J1303-631 centred at $\alpha=13^{\mathrm{h}} 03^{\mathrm{m}} 0.40^{\mathrm{s}}, \delta=-63^{\circ} 11^{\prime} 11.55^{\prime \prime}$, and performed in the $1.384 \mathrm{GHz}$ and $2.368 \mathrm{GHz}$ bands. The archival data used in the analysis, taken as part of the Reinfrank et al. project C1557, are presented in Table 1. Only the archival data taken with all 6 antennas and with observational time longer than 100 minutes were used in the analysis.

\subsection{Data Analysis}

The data analysis was performed using the miriad package (6). The resulting clean radio image at $5.5 \mathrm{GHz}$ is shown in Fig. 1. The pulsar PSR J1301-6305 is detected at the position $\alpha=13^{\mathrm{h}} 01^{\mathrm{m}} 45.680^{\mathrm{s}} \pm 0.012^{\mathrm{s}}, \delta=-63^{\circ} 05^{\prime} 34.848^{\prime \prime} \pm 0.196^{\prime \prime}$. No significant extended emission coincident with the pulsar position was detected. The fitted image root mean square (RMS) noise is calculated using the imsad task at the level of $6.072 \times 10^{-6} \mathrm{Jy} / \mathrm{beam}$ and the major and minor axes of the beam are estimated to be $3.793^{\prime \prime}$ and $3.652^{\prime \prime}$, respectively.

There is no extended emission coincident with the pulsar position detected at $7.5 \mathrm{GHz}$ as well. The fitted image RMS noise, calculated using the imsad task, is at the level of $6.314 \times$ $10^{-6} \mathrm{Jy} / \mathrm{beam}$ and the major and minor axes of the beam are estimated to be $3.055^{\prime \prime}$ and $2.900^{\prime \prime}$, respectively.

The $1.384 \mathrm{GHz}$ (Fig. 2) and $2.368 \mathrm{GHz}$ flux maps which combine all the archival data listed in Table 1 also do not reveal any significant emission coincident with the pulsar. The observations at $1.384 \mathrm{GHz}$, however, reveal a shell-like structure to the east of the pulsar position (Fig. 2) which might be an SNR. The SNR FoV was fit with the two-component model of the point-source and a circular disk using the miriad task imfit (Fig. 2). The point-source component was used to subtract the emission from a source $\sim 3^{\prime}$ to the north from the SNR candidate centre (blue cross on Fig. 2). This point-like source features a $\sim 10$ times higher peak flux than anywhere else in the SNR candidate and is, thus, probably a separate source. The disk fit results in a total integrated flux of $0.692 \pm 0.005 \mathrm{Jy}$ with a peak value of $(6.87 \pm 0.17) \times 10^{-4} \mathrm{Jy} / \mathrm{beam}$. The diameter of the disk is estimated to be $1063^{\prime \prime} \pm 4^{\prime \prime}$. The border of the disk is shown as a blue circle in Fig. 2 with its center marked as a blue cross. The coordinates of the disk center are $\alpha=13^{\mathrm{h}} 04^{\mathrm{m}} 29.059^{\mathrm{s}} \pm 0.035^{\mathrm{s}}$ and $\delta=$ $-63^{\circ} 01^{\prime} 22.140^{\prime \prime} \pm 0.926^{\prime \prime}$. The fitted image RMS noise is estimated to be $3.383 \times 10^{-6} \mathrm{Jy} / \mathrm{beam}$ and major and minor axes of the beam are estimated to be $29.33^{\prime \prime}$ and $26.49^{\prime \prime}$, respectively. 


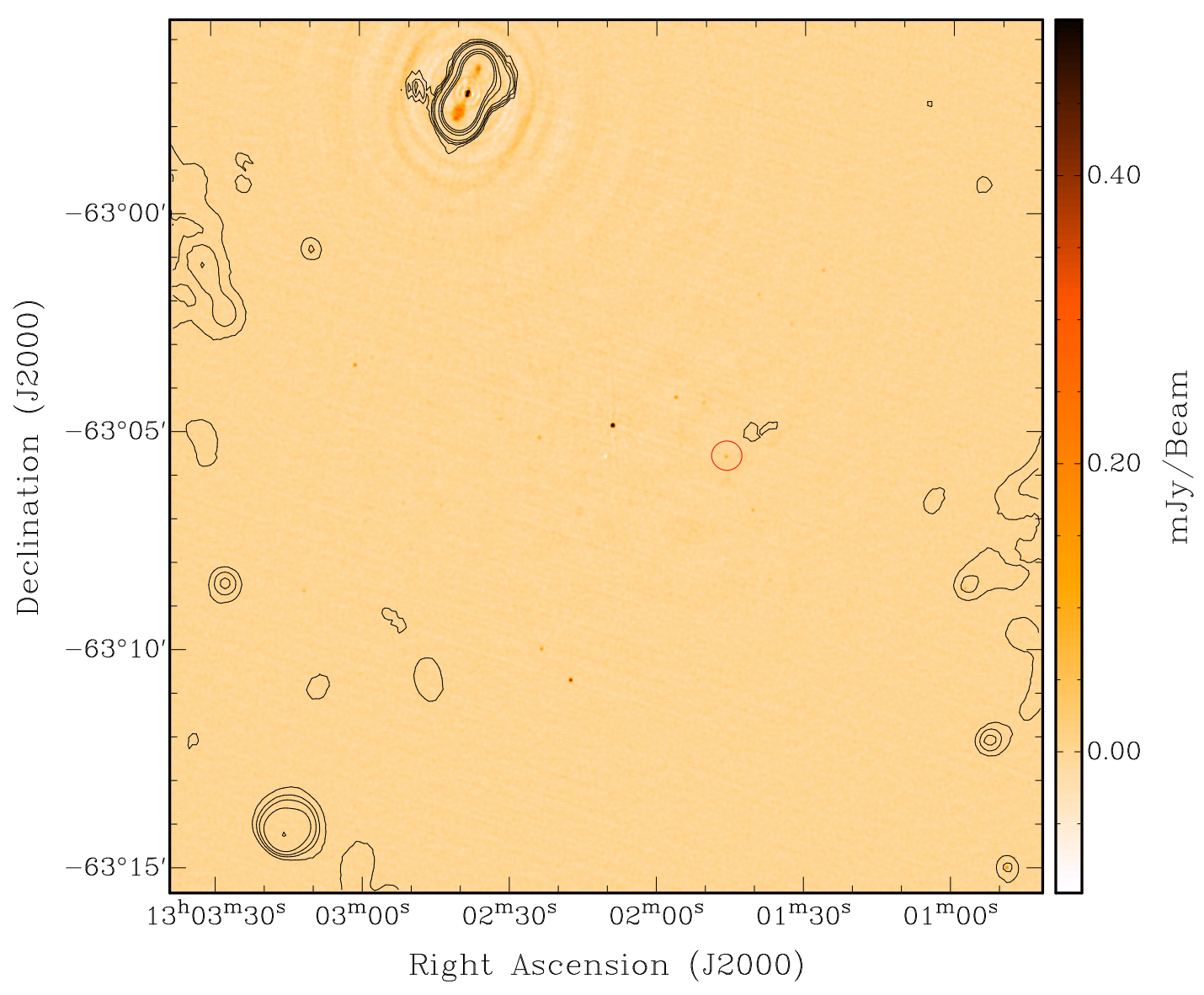

Figure 1: Radio flux map of the HESS J1303-631 FOV at $5.5 \mathrm{GHz}$ overlaid with significance contours from the $1.384 \mathrm{GHz}$ band (from $2 \sigma$ to $6 \sigma$ with the interval of $2 \sigma$ and from $10 \sigma$ to $70 \sigma$ with the interval of $20 \sigma$ ). The red circle indicates the position of the pulsar PSR J1301-6305.

Identification of the detected shell-like structure as an SNR is supported by infrared and optical observations which do not show any extended thermal emission from that region, suggesting that the observed radio emission is non-thermal as expected for SNRs. Figure 3 shows an infrared image obtained from The Two Micron All-Sky Survey (2MASS) in the H-band $(1.65 \mu \mathrm{m})(7)$ overlaid with $1.384 \mathrm{GHz}$ contours. Other infrared surveys as well as optical surveys at various bands do not show anything extra.

\section{Discussion}

\subsection{Implications of the lack of radio emission on the PWN evolution}

The lack of detection of a radio counterpart of the evolved PWN HESS J1303-631 does not allow to draw unambiguous conclusions and leaves plenty of space for speculations. The main uncertainty is the size of the putative radio PWN which might be larger than the FoV which prevents a detection. Below we examine two extreme scenarios which can lead to the non-detection of a radio counterpart. 


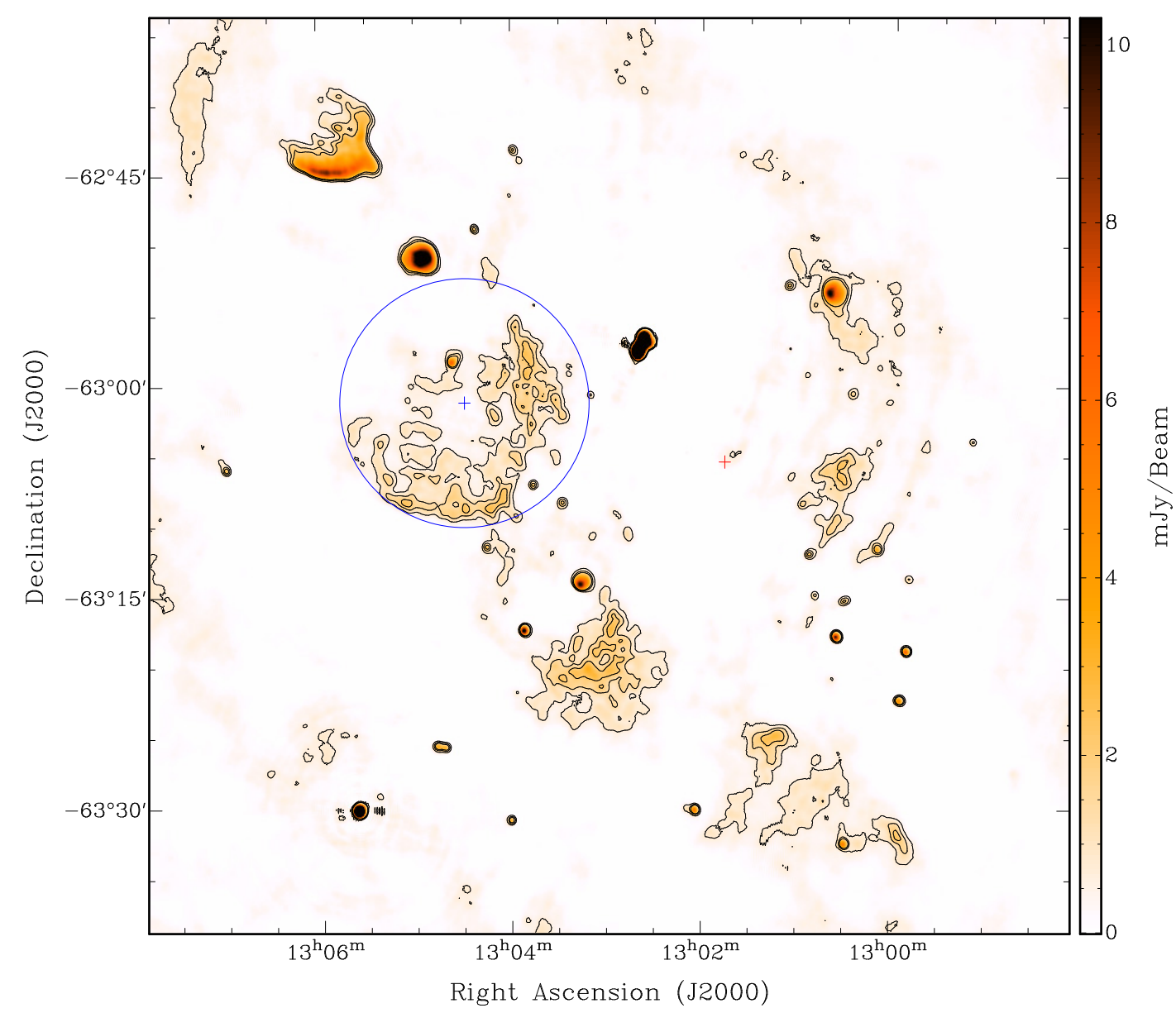

Figure 2: Radio flux map of the HESS J1303-631 FOV at 1.384 GHz. Contour levels represent $2 \sigma, 4 \sigma$, and $6 \sigma 1.384 \mathrm{GHz}$ emission. The red cross indicates the position of the pulsar PSR J1301-6305. The blue circle shows the result of the disk fit to the SNR candidate and the blue cross indicates the centre of the disk.

The size of the $\mathrm{GeV}$ emission region (5) indicates that a high amount of relatively low energy relativistic electrons is spread out to large distances from the pulsar. The same electrons are also responsible for the generation of the radio emission via the synchrotron mechanism (see e.g. 9, and references therein). The radio spectrum of the PWN is expected to follow the same shape as the $\mathrm{GeV}$ spectrum in the energy range where it can be well described by a power-law. Using only two lower energy data points of the GeV spectrum (see Fig. 8 in 5) one can estimate a photon index of $1.01 \pm 0.44_{\text {stat }} \pm 0.71_{\text {syst }}$ which corresponds to an electron spectral index of $p=1.02$ which is well compatible with typical values of $1.0-1.3$ for the electron population responsible for the radio emission in PWNe (e.g. 9; 10). Therefore, it can be expected that the radio emission region should be at least as big as the $\mathrm{GeV}$ emission region, i.e. with an extension of $0.9^{\circ}$ (5). In this case the putative radio PWN would be larger than the $5.5 / 7.5 \mathrm{GHz}$ FoV and comparable to the $1.384 / 2.368 \mathrm{GHz}$ FoV and, thus, the emission from the PWN cannot be detected in observations reported in this paper.

On the other hand, it is expected that the magnetic field is amplified in the vicinity of the pulsar being frozen into the outflowing wind, while at large distances from the pulsar the magnetic field 


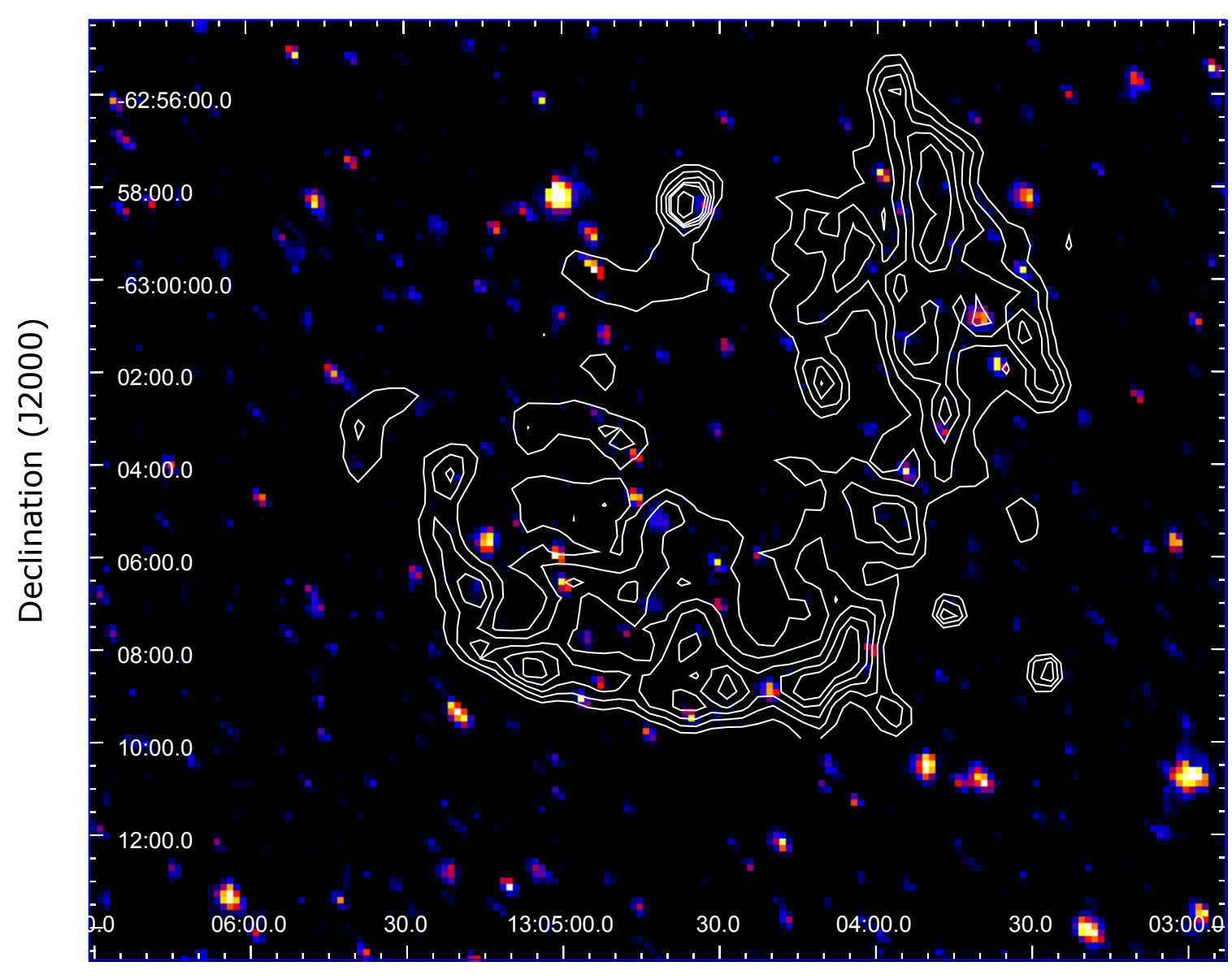

Right Ascension (J2000)

Figure 3: The infrared image of the SNR candidate FoV obtained from the 2MASS survey in the H-band $(1.65 \mu \mathrm{m})(7)$ overlaid with $1.384 \mathrm{GHz}$ radio contours.

can be already relaxed with strength values comparable to the interstellar medium magnetic field of about $3 \mu \mathrm{G}$ supressing the synchrotron emission. In this case the size of the putative radio PWN is defined by the region of higher magnetic field and not by the existence of relativistic electrons. Assuming the size of the X-ray PWN is completely defined by the region of the high magnetic field one might expect a putative radio PWN to be of the same size. This is, however, an extreme case as the size of the X-ray PWN might be also limited by the existence of the very high energy electrons which have a short lifetime and are expected to be only in the immediate vicinity of the pulsar. Therefore, this size should be treated as a lower limit for the region of the higher magnetic field. Assuming the size of the X-ray PWN as reported in (2) the flux upper limit on the radio flux at $5.5 \mathrm{GHz}$ was estimated using a bias-corrected bootstrap method (see e.g. 11) based on the measured counts in the region of the X-ray PWN and performing 20.000 simulations. The upper limit at $99 \%$ confidence level is estimated to be $0.2 \mathrm{mJy}$. This upper limit is much more constraining (by two orders of magnitude) than the one reported in (2) for the insignificant radio feature of a similar size detected at $4.85 \mathrm{GHz}$. Assuming that the same electrons produce radio radiation via the synchrotron mechanism and $\mathrm{GeV}$ emission via inverse Compton (IC) scattering on the Cosmic 
Microwave Background (CMB) and assuming that the magnetic field is uniform across the putative radio PWN one can estimate an upper limit on the magnetic field. For these calculations the total flux in the $\mathrm{GeV}$ range was scaled to the region of the size of the X-ray PWN with extension of $\sim 176^{\prime \prime}(2)$ assuming that the $\mathrm{GeV}$ emission is uniformly distributed. This yields an upper limit of $500 \mu \mathrm{G}$ for assumed $p=1.02$. Similarly one can calculate the magnetic field strength comparing $\mathrm{X}$-ray and $\mathrm{TeV}$ emission. In this case we also assume that $\mathrm{X}$-ray and gamma-ray emission is produced by the same population of electrons the energy distribution of which follows a simple power-law. The total $\mathrm{TeV}$ flux is scaled to the region size of the X-ray PWN and two different values of the electron spectral index are used $p=3.0$ and $p=3.88$ basing on the estimates of the photon indices of the X-ray, $\Gamma=2.0_{-0.7}^{+0.6}$, and $\mathrm{TeV}, \Gamma=2.44 \pm 0.03$, spectra (2). The obtained values of the magnetic field strength are $10 \mu \mathrm{G}$ and $15 \mu \mathrm{G}$, respectively. This approach was checked comparing X-ray flux to the total $\mathrm{TeV}$ flux yielding magnetic field estimates of $1.2 \mu \mathrm{G}$ and $2.8 \mu \mathrm{G}$, respectively, which are compatible with an estimate of $1.4 \mu \mathrm{G}$ obtained in (2). However, it should be noted that a simple scaling of the total $\mathrm{TeV}$ flux is not correct as the $\mathrm{TeV}$ emission features a strong energy-dependent morphology, suggesting that there are much more high energy electrons in the immediate vicinity of the pulsar. This fact would decrease the estimate of the magnetic field strength. Nevertheless, these estimates are in agreement with the non-detection of the radio emission for the assumed electron spectral index $p=1.02$. For further investigation of the spectral energy distribution of the source deep observations in the $\mathrm{TeV}$ band are extremely important as they might give an opportunity to quantitively distinguish spectral properties in different regions of the PWN.

\subsection{The SNR candidate - the birth place of PSR J1301-6305?}

In case the extended source with a shell-like morphology detected at $1.384 \mathrm{GHz}$ is an SNR it could be the birth place of the pulsar PSR J1301-6305. Assuming a distance to the pulsar of $6.6 \mathrm{kpc}$ (2), the angular size of the SNR candidate of 1063" (see above) corresponds to $34 \mathrm{pc}$ in diameter. The Sedov solution (12), which describes the hydrodynamical expansion of an SNR in the adiabatic stage of evolution into the homogeneous medium, provides an estimate of the SNR age for a given size of the remnant

$$
t_{\text {age }}=18\left(\frac{E}{10^{51}[\mathrm{erg}]}\right)^{-2}\left(\frac{n_{\mathrm{ISM}}}{1\left[\mathrm{~cm}^{3}\right]}\right)^{2}\left(\frac{R}{17[\mathrm{pc}]}\right)^{5 / 2}[\mathrm{ky}],
$$

where $E$ is the explosion energy, $n_{\mathrm{ISM}}$ is the number density of the interstellar medium and $R$ is the radius of the remnant. This value is somewhat larger than the characteristic age of the pulsar of $11 \mathrm{ky}$ (13). The characteristic age is defined as an upper limit of the true age of the pulsar under the assumption that the braking index is $n=3$ (spin-down of the pulsar via dipole radiation). It is calculated in a limit of initial spinning period much lower than the current period, $P_{0} \ll P$. However, it is known that the braking index might be considerably lower than 3 (see e.g. 14; 15) which increases the upper limit for the true age of the pulsar. In this case, the true age of the pulsar can be higher than the characteristic age. It should also be noted that the SNR age estimate is strongly dependent on the ambient medium density which is often considerably lower than $1 \mathrm{~cm}^{3}$.

The angular distance between the pulsar and the SNR candidate of $0.32^{\circ}$ corresponds to a projected distance of $36 \mathrm{pc}$ assuming the distance to the pulsar of $6.6 \mathrm{kpc}$. This corresponds to a 
lower limit on the pulsar velocity of $V_{\mathrm{p}} \geq 1000 \mathrm{~km} / \mathrm{s}$ for the estimate of the SNR age presented above. The pulsar is located outside the shell of the SNR candidate, which means that in case the SNR candidate is indeed the birth place of PSR J1301-6305, the pulsar has already escaped the remnant and continues to propagate in the ambient medium. This fact together with the large size of the GeV/TeV PWN (larger than the SNR candidate) suggests that there should be some evidence of distortion of the shell caused by the escape of the pulsar. However, the shell of the SNR candidate does not show strong evidence of distortion on the side of the pulsar. This can be naturally explained if the pulsar is not moving in the projected plane but its velocity has a considerable perpendicular to the projected plane component. In this case the distorted part of the shell is facing the observer and is thus not visible. This configuration, however, requires a higher pulsar velocity depending on the angle $\phi$ between the line of sight and the pulsar proper motion direction

$$
V_{\mathrm{p}}=V_{0} / \sin \phi,
$$

where $V_{0}=1000 \mathrm{~km} / \mathrm{s}$ is the lower limit of the pulsar velocity calculated for $\phi=90^{\circ}$. In any case the association of the pulsar with the SNR candidate would place PSR J1301-6305 among the fastest known pulsars with highest detected velocity of $\sim 1600 \mathrm{~km} / \mathrm{s}(16)$.

\section{References}

[1] F. Aharonian, et al., Serendipitous discovery of the unidentified extended TeV $\gamma$-ray source HESS J1303-631, A\&A, 439 (Sept., 2005) 1013-1021, [a stro-ph/ 0505219 ].

[2] H.E.S.S. Collaboration, A. Abramowski, et al., Identification of HESS J1303-631 as a pulsar wind nebula through $\gamma$-ray, X-ray, and radio observations, A\&A, 548 (Dec., 2012) A46.

[3] O. C. de Jager, et al., Unidentified Gamma-Ray Sources as Ancient Pulsar Wind Nebulae, ArXiv e-prints (June, 2009) [arXiv:0906.2644].

[4] J. J. Condon, M. R. Griffith, and A. E. Wright, The Parkes-MIT-NRAO surveys. IV - Maps for the Southern Survey covering delta in the range -88 to -37 deg, AJ, 106 (Sept., 1993) $1095-1100$.

[5] F. Acero, et al., Constraints on the Galactic Population of TeV Pulsar Wind Nebulae Using Fermi Large Area Telescope Observations, ApJ, 773 (Aug., 2013) 77, [arXiv:1306.5735].

[6] R. J. Sault, P. J. Teuben, and M. C. H. Wright, A Retrospective View of MIRIAD, in Astronomical Data Analysis Software and Systems IV (R. A. Shaw, H. E. Payne, and J. J. E. Hayes, eds.), vol. 77 of Astronomical Society of the Pacific Conference Series, p. 433, 1995. astro-ph/0612759.

[7] M. F. Skrutskie, et al., The Two Micron All Sky Survey (2MASS), AJ, 131 (Feb., 2006) 1163-1183. 
[8] A. E. Wright, et al., The Parkes-MIT-NRAO (PMN) surveys. 2: Source catalog for the southern survey (delta greater than -87.5 deg and less than -37 deg), ApJS, 91 (Mar., 1994) 111-308.

[9] M. J. Vorster, et al., Time-dependent Modeling of Pulsar Wind Nebulae, ApJ, 773 (Aug., 2013) 139, [arXiv:1309.7137].

[10] K. W. Weiler and N. Panagia, Are Crab-type Supernova Remnants (Plerions) Short-lived?, $A \& A, 70$ (Nov., 1978) 419.

[11] J. Carpenter and J. Bithell, Bootstrap confidence intervals: when, which, what? A practical guide for medical statisticians., Statist. Med. 19 (2000) 1141-1164.

[12] L. I. Sedov, Similarity and Dimensional Methods in Mechanics. 1959.

[13] R. N. Manchester, et al., The Australia Telescope National Facility Pulsar Catalogue, AJ, 129 (Apr., 2005) 1993-2006, [astro-ph/ 0412641$].$

[14] M. A. Alpar and A. Baykal, Pulsar braking indices, glitches and energy dissipation in neutron stars, MNRAS, 372 (Oct., 2006) 489-496, [a stro-ph / 0608055 ].

[15] C. M. Espinoza, et al., The Braking Index of PSR J1734-3333 and the Magnetar Population, ApJ, 741 (Nov., 2011) L13, [arXiv:1109.2740].

[16] J. M. Cordes and D. F. Chernoff, Neutron Star Population Dynamics. II. Three-dimensional Space Velocities of Young Pulsars, ApJ, 505 (Sept., 1998) 315-338, [astro-ph/9707308]. 